\title{
Preface of STACS 2016 Special Issue
}

\author{
Christoph Dürr ${ }^{1} \cdot$ Heribert Vollmer $^{2}$
}

Published online: 4 December 2017

(c) Springer Science+Business Media, LLC, part of Springer Nature 2017

This special issue contains seven articles which are based on extended abstracts that were presented at the 33th Symposium on Theoretical Aspects of Computer Science (STACS), which was held at the Université d'Orléans, France from February 17th to 20th, 2016. These extended abstracts were among the top papers of those that were chosen for presentation at STACS 2016 in a highly competitive peer-review process (after which only 54 papers out of 199 submission were accepted).

Compared with the original extended abstracts, the articles have been extended by full proofs and additional results. They underwent a further rigorous reviewing process, following the TOCS standard, completely independent from the selection process of STACS 2016.

The topics of the papers cover data structures, approximation algorithms, compressed data, languages and logic.

The article Testing Shape Restrictions of Discrete Distributions by Clément Louis Canonne, Ilias Diakonikolas, Themis Goulekakis and Ronitt Rubinfeld concerns testing properties of distributions. Fix some property $\mathcal{P}$, like for example log-concavity. Now given some distribution $D$ decide whether $D \in \mathcal{P}$ or $\ell_{a}(D, \mathcal{P})>\epsilon$. The paper provides a general query efficient algorithm which works for a wide range of properties, together with a generic lower bound technique, showing near optimality.

Christoph Dürr

Christoph.Durr@lip6.fr

Heribert Vollmer

vollmer@thi.uni-hannover.de

1 Sorbonne Universités, UPMC Univ Paris 06, CNRS, LIP6, Paris, France

2 Leibniz Universität Hannover, Hanover, Germany 
The article A randomized polynomial kernel for Subset Feedback Vertex Set by Eva-Maria C. Hols and Stefan Kratsch studies the following general problem: Given a graph, and some vertex set $S$ and an integer $k$, the question is whether it is possible to delete $k$ vertices such that no cycle intersects $S$. Since 2011 this problem was known to be fixed parameter tractable in $k$ and people wondered if it admits a polynomial kernelization, which could be randomized. This paper answers the question positively.

The paper Faster Algorithms for the Constrained k-means Problem by Anup Bhattacharya, Ragesh Jaiswa and Amit Kumar study a variant to the classical $k$-means problem. Usually you are given a set of points which you need to partition into $k$ clusters and identify a center for each so to minimize the total squared distance from every point to the center of its cluster. In the present studied version some constraints specify which point sets can be clusters. This is done in an adversarial manner: the algorithm has to produce a list of $\ell k$-centers such that for any clustering one of the alternatives is a $(1+\epsilon)$-approximation. How big must $\ell$ be? The paper improves over a result from SODA'2015, and gives almost close upper and lower bounds.

The paper Catalytic space: non-determinism and hierarchy by Harry Buhrman, Michal Koucký, Bruno Loff and Florian Speelman studies a computation model introduced in STOC'2014. In addition to the normal log-space size working memory we are given a polynomial size auxiliary memory which however needs to be restored by the end of the computation. It was known that the deterministic version of this model is in the class ZPP. The paper shows that this holds also for the nondeterministic version. In addition they show that it is closed under complement and provide hierarchy theorems.

In Size-Treewidth Tradeoffs for Circuits Computing the Element Distinctness Function, Mateus de Oliveira Oliveira considers (variants of) the element distinctness function from a circuit complexity point of view. His results, considering a trade-off between size and treewidth of the circuit, can be seen as a generalization of the work of Neciporuk from 1966 for the case of treewidth 1, i.e., Boolean formulas. Further results address lower bounds for read-once circuits.

The paper Efficiently Finding All Maximal $\alpha$-gapped Repeats by Pawel Gawrychowski, Tomohiro I, Shunsuke Inenaga, Dominik Köppl and Florin Manea studies gapped repeats, repetitive structures in words which are studied intensively in the area of combinatorics on words. The authors first prove an upper bound on the number of maximal gapped repeats and then use this to develop an optimal agorithm to find such repeats.

The final contribution in this special issue is the paper Knapsack in Graph Groups by Markus Lohrey and Georg Zetzsche. The problem addressed is the following: Fix a finitely generated group $G$. Given now group elements $g_{1}, \ldots, g_{k}, g$ (all given by finite words over the generators), the question is if the equalition $g_{1}^{x_{1}} \ldots g_{k}^{x_{k}}=g$ has a solution. If the group is the group of the integers, this is essentially Karp's classical knapsack problem. Here, so called graph groups are studied and the complexity of the problem in two variants, compressed (where the group elements are represented by straight line programs) and uncompressed (where group elements are explicitly given 
by words over generators), is determined. This paper presents and extends results from two extended abstracts by the authors, one was presented at the 33th Symposium on Theoretical Aspects of Computer Science 2016 in Orléans and one at the 34th Symposium on Theoretical Aspects of Computer Science 2017 in Hannover.

We thank the authors for submitting their papers to this issue, and we thank the referees for their thorough reviews of the manuscripts.

Christoph Dürr and Heribert Vollmer, Guest Editors, November 2017 